\title{
EXAMPLES OF FINITE-DIMENSIONAL POINTED HOPF ALGEBRAS IN CHARACTERISTIC $2^{\dagger}$
}

\author{
NICOLÁS ANDRUSKIEWITSCH \\ FAMAF-Universidad Nacional de Córdoba, CIEM (CONICET), \\ Medina Allende s/n, Ciudad Universitaria, (5000) Córdoba, República Argentina \\ e-mail:andrus@famaf.unc.edu.ar
}

\author{
DIRCEU BAGIO, SARADIA DELLA FLORA and DAIANA FLÔRES \\ Departamento de Matemática, Universidade Federal de Santa Maria, \\ 97105-900, Santa Maria, RS, Brazil \\ e-mails: bagio@smail.ufsm.br; saradia.flora@ufsm.br; flores@ufsm.br
}

(Received 16 May 2020; revised 8 October 2020; accepted 30 October 2020)

\begin{abstract}
We present new examples of finite-dimensional Nichols algebras over fields of characteristic 2 from braided vector spaces that are not of diagonal type, admit realizations as Yetter-Drinfeld modules over finite abelian groups, and are analogous to Nichols algebras of finite Gelfand-Kirillov dimension in characteristic 0 . New finite-dimensional pointed Hopf algebras over fields of characteristic 2 are obtained by bosonization with group algebras of suitable finite abelian groups.
\end{abstract}

2020 Mathematics Subject Classification. Primary: 16T20; Secondary: 17B37

1. Introduction. The goal of this paper is to present new examples of finitedimensional Hopf algebras in characteristic 2, which are pointed, non-commutative, and non-cocommutative. Following the usual guidelines of the lifting method, we focus on finite-dimensional Nichols algebras, then the Hopf algebras are obtained routinely by bosonization. The main result of [2] (in characteristic 0) is the classification of the Nichols algebras with finite Gelfand-Kirillov dimension arising from braided vector spaces $(V, c)$ that decompose as:

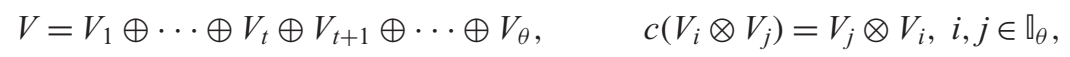

where $V_{1}, \ldots, V_{t}$ are blocks (see Section 2.2); $V_{t+1}, \ldots, V_{\theta}$ are points (i.e. have dimension 1); and the braidings have a specific form, see for example, (3.2), (5.2). This result relies on the classification in [7] and assumes a Conjecture treated partially in [3], both about Nichols algebras of diagonal type. However in positive characteristic, the

\footnotetext{
$\dagger$ This material is based upon work supported by the National Science Foundation under Grant No. DMS1440140, while N. A. was in residence at the Mathematical Sciences Research Institute in Berkeley, California, in the Spring 2020 semester. The work of N. A. was partially supported by CONICET, Secyt (UNC), and the Alexander von Humboldt Foundation through the Research Group Linkage Programme.
} 
Table 1. Finite-dimensional Nichols algebra in characteristic 2.

\begin{tabular}{lccc}
\hline$V$ & $\mathscr{B}(V)$ & $\operatorname{dim} K$ & $\operatorname{dim} \mathscr{B}(V)$ \\
\hline $\mathfrak{L}_{\wp}(1,1)$ & Proposition 3.6 & $2^{3}$ & $2^{7}$ \\
$\mathfrak{L}_{\wp}(1, a), a \neq 1$ & Proposition 3.7 & $2^{4}$ & $2^{8}$ \\
$\mathfrak{P}(\mathbf{q}, \mathbf{a}), \mathbf{a} \in\left(\mathbb{k}^{\times}\right)^{t}$ & Proposition 5.4 & $2^{|\mathcal{A}|}$ & $2^{4 t+|\mathcal{A}|}$ \\
$\mathfrak{E}_{\wp}(1)$ & Proposition 6.2 & $2^{2}$ & $2^{4}$ \\
$\mathfrak{E}_{\wp}(\omega), \omega \in \mathbb{G}_{3}^{\prime}$ & Proposition 6.3 & $3^{3}$ & $2^{2} 3^{3}$ \\
\hline
\end{tabular}

classification of finite-dimensional Nichols algebras of diagonal type is known only in rank $\leq 4[9,10,11]$. Inspired by [6] and by familiar phenomena in Lie theory in positive characteristic, examples of finite-dimensional Nichols algebras in odd characteristic were constructed in [4] by analogy with the Nichols algebras in [2] that have infinite dimension. Here we extend these constructions assuming that the base field $\mathbb{k}$ is algebraically closed of characteristic 2 . There are new features as $1=-1$ now. For instance in characteristic 0 , two main actors are the Jordan and the super Jordan planes. Their restricted versions in characteristic $p>2$ have dimensions $p^{2}[6]$ and $4 p^{2}$ [4], respectively. When char $\mathbb{k}=2$, they merge in the restricted Jordan plane that has dimension $16=4 \times 2^{2}$ [6]. Other families of [2] also merge. Finally, the fact that $x_{i}^{2}=0$ for suitable $x_{i}$ in the braided vector space brings on more examples with finite dimension. Let us present the main result of this paper.

THEOREM. If $V$ is a braided vector space as in Table 1, then the dimension of the Nichols algebra $\mathscr{B}(V)$ is finite.

See Section 2.3.2 for the meaning of $K$. The braided vector spaces $\mathfrak{L}_{\wp}(1,1)$ appear to be close to $\mathfrak{L}(-1, \mathscr{G})$ and $\mathfrak{L}_{-1}(-1, \mathscr{G})$ in $\left[4\right.$, Table 1], but $\mathscr{B}\left(\mathfrak{L}_{\wp}(1, a)\right), a \neq 1$ has no finite-dimensional analog in char $\mathbb{k}=p>2$. Similarly, the algebras $\mathscr{B}(\mathfrak{P}(\mathbf{q}, \mathbf{a}))$ are finitedimensional in odd characteristic only when the entries of a belong to the prime field, in contrast with characteristic 2. Also $\mathfrak{E}_{\wp}(\omega)$ does not appear in the loc. cit. Albeit no classification is envisageable yet as the knowledge of diagonal type is still incomplete, we present partial results in Theorems 3.1, 4.1, and 6.1.

After spelling out some preliminaries in Section 2, we devote Sections 3, 4, 5, and 6 to Nichols algebras of one block and one point, one block and several points, several blocks and one point, and one pale block and one point, respectively. Our proofs rely on the splitting technique Section 2.3.2 and the classifications in $[9,10,11]$. Explicit examples of finite-dimensional pointed Hopf algebras are discussed in Sections 3.2, 5.2, and 6.2. More examples by lifting will be presented in a future work.

\section{Preliminaries.}

2.1. Notations and conventions. We denote the natural numbers by $\mathbb{N}, \mathbb{N}_{0}=\mathbb{N} \cup$ $\{0\}$. We set $\rrbracket_{k, \ell}=\left\{n \in \mathbb{N}_{0}: k \leq n \leq \ell\right\}, \rrbracket_{\ell}=\rrbracket_{1, \ell}$ and $\mathbb{N}_{\geq \ell}=\mathbb{N} \backslash \rrbracket_{\ell-1}$, for $k<\ell \in \mathbb{N}_{0}$. We work over an algebraically closed field $\mathbb{k}$ of characteristic 2 . The group of $N$-th roots of unity in $\mathbb{k}$ is denoted by $\mathbb{G}_{N} ; \mathbb{G}_{N}^{\prime}$ is the subset of the primitive roots of order $N$ and $\mathbb{G}_{\infty}=$ $\bigcup_{N \in \mathbb{N}} \mathbb{G}_{N}$.

Throughout $H$ is a Hopf algebra with bijective antipode $\mathcal{S}$. We use the notations $G(H)=$ the group of grouplikes in $H, \mathcal{P}(H)=$ the space of primitive elements, $\widehat{H}=$ $\operatorname{Hom}_{\mathrm{alg}}(H, \mathbb{k}),{ }_{H}^{H} \mathcal{Y} \mathcal{D}=$ the category of Yetter-Drinfeld modules over $H$; see for example $[12,11.6]$. 


\subsection{Yetter-Drinfeld modules.}

\subsubsection{Braided vector spaces}

A braided vector space $V$ is a pair $(V, c)$ where $V$ is a vector space and $c \in G L\left(V^{\otimes 2}\right)$ is a solution of the braid equation:

$$
(c \otimes \mathrm{id})(\mathrm{id} \otimes c)(c \otimes \mathrm{id})=(\mathrm{id} \otimes c)(c \otimes \mathrm{id})(\mathrm{id} \otimes c) .
$$

We are interested in two classes of braided vector spaces. First, $(V, c)$ or simply $V$ is of diagonal type if there exist a basis $\left(x_{i}\right)_{i \in \mathbb{\Xi}_{\theta}}$ of $V$ and a matrix $\mathbf{q}=\left(q_{i j}\right)_{i, j \in \mathbb{1}_{\theta}}$ such that $q_{i j} \in \mathbb{k}^{\times}$ and $c\left(x_{i} \otimes x_{j}\right)=q_{i j} x_{j} \otimes x_{i}$ for all $i, j \in \mathbb{\square}_{\theta}$. We denote in $T(V)$, or any quotient braided Hopf algebra:

$$
x_{i j}=\left(\operatorname{ad}_{c} x_{i}\right) x_{j}, \quad x_{i_{1} i_{2} \ldots i_{M}}=\left(\operatorname{ad}_{c} x_{i_{1}}\right) x_{i_{2} \ldots i_{M}}, \quad i, j, i_{1}, \ldots, i_{M} \in \mathbb{\mathbb { V }}, \quad M \geq 2 .
$$

Second, let $\epsilon \in \mathbb{k}^{\times}$and $\ell \in \mathbb{N}_{\geq 2}$. A block $\mathcal{V}(\epsilon, \ell)$ is a braided vector space with a basis

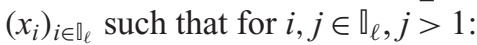

$$
c\left(x_{i} \otimes x_{1}\right)=\epsilon x_{1} \otimes x_{i}, \quad c\left(x_{i} \otimes x_{j}\right)=\left(\epsilon x_{j}+x_{j-1}\right) \otimes x_{i} .
$$

For simplicity, a block $\mathcal{V}(\epsilon, 2)$ of dimension 2 is called an $\epsilon$-block.

\subsubsection{Realizations}

Any Yetter-Drinfeld module $V$ bears a structure of braided vector space by $c(v \otimes w)=$ $v_{(-1)} \cdot w \otimes v_{(0)}, v, w \in V$ where $\delta(v)=v_{(-1)} \otimes v_{(0)}$. The braided vector spaces above appear as Yetter-Drinfeld modules in different ways called realizations. Let $\Gamma$ be an abelian group and let $\widehat{\Gamma}$ be the group of characters of $\Gamma$. The Yetter-Drinfeld modules over the group algebra $\mathbb{k} \Gamma$ are the $\Gamma$-graded $\Gamma$-modules, the $\Gamma$-grading being denoted by $V=\oplus_{g \in \Gamma} V_{g}$; thus $h \cdot V_{g}=V_{g}$ for $g, h \in \Gamma$. If $g \in \Gamma$ and $\chi \in \widehat{\Gamma}$, then the one-dimensional vector space $\mathbb{k}_{g}^{\chi}$, with action and coaction given by $g$ and $\chi$, is in ${ }_{\mathbb{k} \Gamma}^{\mathbb{k} \Gamma} \mathcal{Y} \mathcal{D}$. Given $V \in \mathbb{k}_{\mathrm{k} \Gamma}^{\mathrm{k}} \mathcal{Y} \mathcal{D}$ with a basis $\left(v_{i}\right)_{i \in I}$ where $v_{i}$ is homogeneous of degree $g_{i}$, there are skew derivations $\partial_{i}, i \in I$, of $T(V)$ such that:

$$
\partial_{i}\left(v_{j}\right)=\delta_{i j}, i, j \in I, \quad \partial_{i}(x y)=\partial_{i}(x)\left(g_{i} \cdot y\right)+x \partial_{i}(y), x, y \in T(V) .
$$

More generally, a $Y D$-pair for $H$ is a pair $(g, \chi) \in G(H) \times \widehat{H}$ such that:

$$
\chi(h) g=\chi\left(h_{(2)}\right) h_{(1)} g \mathcal{S}\left(h_{(3)}\right), \quad h \in H .
$$

Let $\mathbb{k}_{g}^{\chi}$ be a one-dimensional vector space with $H$-action and $H$-coaction given by $\chi$ and $g$, respectively; then (2.3) says that $\mathbb{k}_{g}^{\chi} \in{ }_{H}^{H} \mathcal{Y} \mathcal{D}$. Thus, a realization of $V$ of diagonal type with matrix $\mathbf{q}=\left(q_{i j}\right)_{i, j \in \mathbb{D}_{\theta}}$ is just a collection $\left(g_{1}, \chi_{1}\right), \ldots,\left(g_{\theta}, \chi_{\theta}\right)$ such that $q_{i j}=\chi_{j}\left(g_{i}\right)$ for all $i, j \in \mathbb{\square}_{\theta}$.

\subsubsection{Realizations of $\epsilon$-blocks}

For $\chi \in \widehat{H}$, the space of $(\chi, \chi)$-derivations is

$$
\operatorname{Der}_{\chi, \chi}(H, \mathbb{k})=\left\{\eta \in H^{*}: \eta(h \ell)=\chi(h) \eta(\ell)+\chi(\ell) \eta(h) \forall h, \ell \in H\right\} .
$$

The realizations of $\epsilon$-blocks are given by the notion of YD-triple for $H$ [4]; this is a collection $(g, \chi, \eta)$ where $(g, \chi)$, is a YD-pair for $H, \eta \in \operatorname{Der}_{\chi, \chi}(H, \mathbb{k}), \chi(g)=\epsilon$, $\eta(g)=1$ and 


$$
\eta(h) g=\eta\left(h_{(2)}\right) h_{(1)} g \mathcal{S}\left(h_{(3)}\right), \quad h \in H .
$$

Given a YD-triple $(g, \chi, \eta)$, we define $\mathcal{V}_{g}(\chi, \eta) \in_{H}^{H} \mathcal{Y} \mathcal{D}$ as the vector space with a basis $\left(x_{i}\right)_{i \in \mathbb{Z}_{2}}$, whose $H$-action and $H$-coaction are given by:

$$
h \cdot x_{1}=\chi(h) x_{1}, \quad h \cdot x_{2}=\chi(h) x_{2}+\eta(h) x_{1}, \quad \delta\left(x_{i}\right)=g \otimes x_{i}, \quad h \in H, i \in \mathbb{I}_{2} .
$$

Then, $\mathcal{V}_{g}(\chi, \eta) \simeq \mathcal{V}(\epsilon, 2)$ as braided vector spaces.

EXAMPLE 2.1. Let $\epsilon=1$ and $\Gamma=\langle g\rangle$ be a cyclic group of order $N$. Let $\mathcal{V}$ be the vector

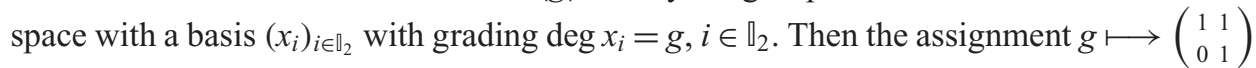
defines a representation of $\Gamma$ (hence, a structure of Yetter-Drinfeld module over $\mathbb{k} \Gamma$ ) if and only if $N$ is even. Thus if $\operatorname{dim} H<\infty$ and $H$ admits a YD-triple (for $\epsilon=1$ ), then $\operatorname{dim} H$ is even.

2.3. Nichols algebras. Let $V \in{ }_{H}^{H} \mathcal{Y D}$. The Nichols algebra of $V$ is the unique graded connected Hopf algebra $\mathscr{B}(V)=\oplus_{n \geq 0} \mathscr{B}^{n}(V)$ in ${ }_{H}^{H} \mathcal{Y D}$ such that $V \simeq \mathscr{B}^{1}(V)=\mathcal{P}(\mathscr{B}(V))$ generates $\mathscr{B}(V)$ as algebra. See [1] for an exposition. The algebra and coalgebra underlying $\mathscr{B}(V)$ depend only on the braiding. If $V \in \in_{\mathbb{k} \Gamma}^{\mathbb{k} \Gamma} \mathcal{Y} \mathcal{D}$ is as in Section 2.2, then the $\partial_{i}$ 's induce skew-derivations on $\mathscr{B}(V)$. Then, $w \in \mathscr{B}^{k}(V), k \geq 1$, is 0 if and only if $\partial_{i}(w)=0$ in $\mathscr{B}(V)$ for all $i \in I$.

\subsubsection{The restricted Jordan plane}

This is the Nichols algebra of a 1-block.

THEOREM $2.2([6])$. The algebra $\mathscr{B}(\mathcal{V}(1,2))$ is presented by generators $x_{1}, x_{2}$ and relations:

$$
x_{1}^{2}, \quad x_{2}^{4}, \quad x_{2}^{2} x_{1}+x_{1} x_{2}^{2}+x_{1} x_{2} x_{1}, \quad x_{1} x_{2} x_{1} x_{2}+x_{2} x_{1} x_{2} x_{1} .
$$

Let $x_{21}:=x_{1} x_{2}+x_{2} x_{1}$. Then, $\operatorname{dim} \mathscr{B}(\mathcal{V}(1,2))=16$ since $\mathscr{B}(\mathcal{V})$ has a basis:

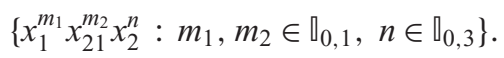

\subsubsection{The splitting technique}

Let $V=V_{1} \oplus V_{2}$ be a direct sum of objects in ${ }_{\mathbb{k} \Gamma}^{\mathbb{k} \Gamma} \mathcal{Y} \mathcal{D}$. Then, $\mathscr{B}(V) \simeq K \# \mathscr{B}\left(V_{1}\right)$ where $K=\mathscr{B}(V)^{\text {co } \mathscr{B}\left(V_{1}\right)}$. By [8, Proposition 8.6], $K$ is the Nichols algebra of

$$
K^{1}=\operatorname{ad}_{c} \mathscr{B}\left(V_{1}\right)\left(V_{2}\right) .
$$

Here, $K^{1} \in \underset{\mathscr{B}\left(V_{1}\right) \# \mathbb{k} \Gamma}{\mathscr{P}\left(V_{1}\right) \# \mathbb{D} \Gamma}$ with the adjoint action and the coaction given by

$$
\delta=\left(\pi_{\mathscr{B}\left(V_{1}\right) \# \mathrm{k} \Gamma} \otimes \mathrm{id}\right) \Delta_{\mathscr{B}(V) \# \mathbb{k} \Gamma} .
$$

3. One block and one point. Let $\left(q_{i j}\right)_{i, j \in \mathbb{D}_{2}}, q_{i j} \in \mathbb{k}^{\times}, a \in \mathbb{k}$. In this section, we assume that

$$
q_{11}=1, \quad q_{12} q_{21}=1
$$


Sometimes, we use $\wp=q_{12}=q_{21}^{-1}$. Let $\mathfrak{L}_{\wp}\left(q_{22}, a\right)$ be the braided vector space with basis $\left(x_{i}\right)_{i \in \mathbb{1}_{3}}$ and braiding given by

$$
\left(c\left(x_{i} \otimes x_{j}\right)\right)_{i, j \in \rrbracket_{3}}=\left(\begin{array}{ccc}
x_{1} \otimes x_{1} & \left(x_{2}+x_{1}\right) \otimes x_{1} & q_{12} x_{3} \otimes x_{1} \\
x_{1} \otimes x_{2} & \left(x_{2}+x_{1}\right) \otimes x_{2} & q_{12} x_{3} \otimes x_{2} \\
q_{21} x_{1} \otimes x_{3} & q_{21}\left(x_{2}+a x_{1}\right) \otimes x_{3} & q_{22} x_{3} \otimes x_{3}
\end{array}\right) .
$$

Let $V_{1}=\left\langle x_{1}, x_{2}\right\rangle \simeq \mathcal{V}(1,2)$ (the block) and $V_{2}=\left\langle x_{3}\right\rangle$ (the point); then $\mathfrak{L}_{\wp}\left(q_{22}, a\right)=V_{1} \oplus$ $V_{2}$. For simplicity, $V=\mathfrak{L}_{\wp}\left(q_{22}, a\right)$. Let $\Gamma=\mathbb{Z}^{2}$ with canonical basis $g_{1}, g_{2}$. Observe that $(V, c)$ can be realized in ${ }_{\mathbb{k} \Gamma}^{\mathbb{k} \Gamma} \mathcal{Y} \mathcal{D}$ via:

$$
\begin{array}{lll}
g_{1} \cdot x_{1}=x_{1}, & g_{1} \cdot x_{2}=x_{1}+x_{2}, & g_{1} \cdot x_{3}=q_{12} x_{3}, \\
g_{2} \cdot x_{1}=q_{21} x_{1}, & g_{2} \cdot x_{2}=q_{21}\left(x_{2}+a x_{1}\right), & g_{2} \cdot x_{3}=q_{22} x_{3}, \\
\operatorname{deg} x_{1}=g_{1}, & \operatorname{deg} x_{2}=g_{1}, & \operatorname{deg} x_{3}=g_{2} .
\end{array}
$$

If $a=0$, then $\mathscr{B}\left(\mathfrak{L}_{\wp}\left(q_{22}, 0\right)\right) \simeq \mathscr{B}\left(V_{1}\right) \underline{\otimes} \mathscr{B}\left(V_{2}\right)$, where $\underline{\otimes}$ is the braided tensor product. Since $\operatorname{dim} \mathscr{B}\left(V_{1}\right)=2^{4}, \operatorname{dim} \mathscr{B}\left(\mathfrak{L}_{\wp}\left(q_{22}, 0\right)\right)<\infty \Longleftrightarrow \operatorname{dim} \mathscr{B}\left(\mathbb{k} x_{3}\right)<\infty \Longleftrightarrow q_{22} \in \mathbb{G}_{\infty}$. Thus, we can assume that $a \in \mathbb{k}^{\times}$.

Our main goal in this section is to prove the following result.

Theorem 3.1. Assume (3.1) and that $a \neq 0$. Then, $\operatorname{dim} \mathscr{B}\left(\mathfrak{L}_{\wp}\left(q_{22}, a\right)\right)<\infty$ if and only if $q_{22}=1$. Precisely, $\operatorname{dim} \mathscr{B}\left(\mathfrak{L}_{\wp}(1, a)\right)= \begin{cases}2^{7} & \text { if } a=1, \\ 2^{8} & \text { if } a \in \mathbb{k} \backslash\{0,1\} .\end{cases}$

We shall apply the splitting technique cf. Section 2.3.2. To describe $K^{1}$, we set

$$
z_{n}:=\left(\operatorname{ad}_{c} x_{2}\right)^{n} x_{3}, \quad n \in \mathbb{N}_{0} .
$$

We establish first a series of useful formulae.

LEMMA 3.2. The following formulae hold in $\mathscr{B}(V)$ for all $n \in \mathbb{N}_{0}$ :

$$
\begin{array}{lll}
g_{1} \cdot z_{n}=q_{12} z_{n}, & x_{1} z_{n}=q_{12} z_{n} x_{1}, & x_{21} z_{n}=q_{12}^{2} z_{n} x_{21}, \\
g_{2} \cdot z_{n}=q_{21}^{n} q_{22} z_{n}, & x_{2} z_{n}=q_{12} z_{n} x_{2}+z_{n+1} . &
\end{array}
$$

Proof. Note that (3.5) holds for $n=0$. Indeed, $g_{1} \cdot z_{0}=g_{1} \cdot x_{3}=q_{12} z_{0}$ and using derivations is easy to check that $x_{1} z_{0}=q_{12} z_{0} x_{1}$ and $x_{21} z_{0}=q_{12}^{2} z_{0} x_{21}$. Now suppose that (3.5) holds for $n$. Then, $z_{n+1}=\left(\operatorname{ad}_{c} x_{2}\right)^{n+1} x_{3}=\left(\operatorname{ad}_{c} x_{2}\right) z_{n}=x_{2} z_{n}+\left(g_{1} \cdot z_{n}\right) x_{2}=x_{2} z_{n}+q_{12} z_{n} x_{2}$. So we compute

$$
\begin{aligned}
g_{1} \cdot z_{n+1} & =g_{1} \cdot\left(x_{2} z_{n}+q_{12} z_{n} x_{2}\right)=q_{12}\left(x_{1}+x_{2}\right) z_{n}+q_{12}^{2} z_{n}\left(x_{1}+x_{2}\right) \\
& =q_{12}\left[\left(x_{2} z_{n}+q_{12} z_{n} x_{2}\right)+\left(x_{1} z_{n}+q_{12} z_{n} x_{1}\right)\right]=q_{12} z_{n+1} .
\end{aligned}
$$

Similarly,

$$
\begin{aligned}
x_{1} z_{n+1} & =x_{1}\left(x_{2} z_{n}+q_{12} z_{n} x_{2}\right)=\left(x_{21}+x_{2} x_{1}\right) z_{n}+q_{12}^{2} z_{n} x_{1} x_{2} \\
& =q_{12}^{2} z_{n} x_{21}+q_{12} x_{2} z_{n} x_{1}+q_{12}^{2} z_{n}\left(x_{21}+x_{2} x_{1}\right) \\
& =q_{12}\left(x_{2} z_{n}+q_{12} z_{n} x_{2}\right) x_{1}=q_{12} z_{n+1} x_{1} .
\end{aligned}
$$


Also, since $x_{21} x_{2}=x_{2} x_{21}+x_{21} x_{1}$ we have that

$$
\begin{aligned}
x_{21} z_{n+1} & =x_{21}\left(x_{2} z_{n}+q_{12} z_{n} x_{2}\right)=x_{21} x_{2} z_{n}+q_{12}^{3} z_{n} x_{21} x_{2} \\
& =x_{2} x_{21} z_{n}+x_{21} x_{1} z_{n}+q_{12}^{3} z_{n} x_{21} x_{2} \\
& =q_{12}^{2} x_{2} z_{n} x_{21}+q_{12} x_{21} z_{n} x_{1}+q_{12}^{3} z_{n}\left(x_{2} x_{21}+x_{21} x_{1}\right) \\
& =q_{12}^{2} x_{2} z_{n} x_{21}+q_{12}^{3} z_{n} x_{21} x_{1}+q_{12}^{3} z_{n} x_{2} x_{21}+q_{12}^{3} z_{n} x_{21} x_{1} \\
& =q_{12}^{2}\left(x_{2} z_{n}+q_{12} z_{n} x_{2}\right) x_{21} \\
& =q_{12}^{2} z_{n+1} x_{21} .
\end{aligned}
$$

Finally, the first equation in (3.6) follows by induction. For $n=0, g_{2} \cdot z_{0}=q_{22} z_{0}$. Suppose that $g_{2} \cdot z_{n}=q_{21}^{n} q_{22} z_{n}$. Then,

$$
\begin{aligned}
g_{2} \cdot z_{n+1} & =g_{2} \cdot\left(x_{2} z_{n}+q_{12} z_{n} x_{2}\right) \\
& =q_{21}\left(x_{2}+a x_{1}\right)\left(q_{21}^{n} q_{22} z_{n}\right)+q_{12}\left(q_{21}^{n} q_{22} z_{n}\right) q_{21}\left(x_{2}+a x_{1}\right) \\
& =q_{21}^{n+1} q_{22}\left(x_{2} z_{n}+q_{12} z_{n} x_{2}\right)+a q_{21}^{n+1} q_{22}\left(x_{1} z_{n}+q_{12} z_{n} x_{1}\right) \\
& =q_{21}^{n+1} q_{22} z_{n+1} .
\end{aligned}
$$

We define

$$
\begin{aligned}
\mu_{0}=1, & \mu_{1}=a, & \mu_{2}=a, & \mu_{3}=a(a+1), \\
y_{0}=1, & y_{1}=x_{1}, & y_{2}=x_{21}, & y_{3}=x_{1} x_{21} .
\end{aligned}
$$

LeMmA 3.3. For all $k \in \mathbb{N}_{0}, \partial_{1}\left(z_{k}\right)=\partial_{2}\left(z_{k}\right)=0$, and

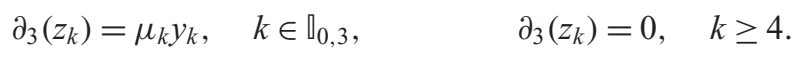

Proof. Clearly, $\partial_{1}\left(z_{0}\right)=\partial_{2}\left(z_{0}\right)=0, \partial_{3}\left(z_{0}\right)=1$. Recursively, $\partial_{1}\left(z_{k}\right)=0$ for all $k$. If $\partial_{2}\left(z_{k}\right)=0$, then $\partial_{2}\left(z_{k+1}\right)=\partial_{2}\left(x_{2} z_{k}+q_{12} z_{k} x_{2}\right)=g_{1} \cdot z_{k}+q_{12} z_{k} \stackrel{(3.5)}{=} 0$. Next,

$$
\begin{aligned}
\partial_{3}\left(z_{1}\right) & =\partial_{3}\left(x_{2} x_{3}+q_{12} x_{3} x_{2}\right)=x_{2}+q_{12}\left(q_{21}\left(x_{2}+a x_{1}\right)\right)=a x_{1}=\mu_{1} y_{1}, \\
\partial_{3}\left(z_{2}\right) & =\partial_{3}\left(x_{2} z_{1}+q_{12} z_{1} x_{2}\right)=a x_{2} x_{1}+q_{12} a x_{1} q_{21}\left(x_{2}+a x_{1}\right)=a x_{21}=\mu_{2} y_{2}, \\
\partial_{3}\left(z_{3}\right) & =\partial_{3}\left(x_{2} z_{2}+q_{12} z_{2} x_{2}\right)=a x_{2} x_{21}+q_{12} a x_{21} q_{21}\left(x_{2}+a x_{1}\right) \\
& =a x_{2}\left(x_{2} x_{1}+x_{1} x_{2}\right)+a\left(x_{2} x_{1}+x_{1} x_{2}\right)\left(x_{2}+a x_{1}\right) \\
& =a x_{2}^{2} x_{1}+a x_{1} x_{2}^{2}+a^{2} x_{1} x_{2} x_{1} \stackrel{(2.5)}{=}\left(a+a^{2}\right) x_{1} x_{2} x_{1} \\
& =\left(a+a^{2}\right) x_{1} x_{21}=\mu_{3} y_{3}, \\
\partial_{3}\left(z_{4}\right) & =\partial_{3}\left(x_{2} z_{3}+q_{12} z_{3} x_{2}\right) \\
& =\left(a+a^{2}\right) x_{2} x_{1} x_{2} x_{1}+q_{12}\left(a+a^{2}\right) x_{1} x_{2} x_{1}\left(g_{2} \cdot x_{2}\right) \\
& =\left(a+a^{2}\right) x_{2} x_{1} x_{2} x_{1}+q_{12}\left(a+a^{2}\right) x_{1} x_{2} x_{1}\left(q_{21}\left(x_{2}+a x_{1}\right)\right) \\
& =\left(a+a^{2}\right)\left(x_{2} x_{1} x_{2} x_{1}+x_{1} x_{2} x_{1} x_{2}\right) \stackrel{(2.5)}{=} 0 .
\end{aligned}
$$


Lemma 3.4. Let $B_{1}:=\left\{z_{i}: i \in \mathbb{\square}_{0,2}\right\}$ and $B_{2}:=\left\{z_{i}: i \in \mathbb{\square}_{0,3}\right\}$. If $a=1$ (resp. $a \neq 1$ ), then $B_{1}$ (resp. $\left.B_{2}\right)$ is a basis of $K^{1}$.

Proof. Notice that $\left(\operatorname{ad}_{c} x_{1}\right) z_{n}=0$ and $\left(\operatorname{ad}_{c} x_{21}\right) z_{n}=0$. By Theorem 2.2 and Lemma 3.3, if $a=1$ (resp. $a \neq 1$ ), then $B_{1}$ (resp. $\left.B_{2}\right)$ generates $K^{1}$. Since the elements of $B_{i}\left(i \in \mathbb{\square}_{0,2}\right)$ are homogeneous of distinct degrees and are nonzero, it follows that $B_{i}\left(i \in \mathbb{Z}_{0,2}\right)$ is a linearly independent set.

Let $i \in \mathbb{N}_{0}$. We define recursively the scalars $v_{i, j}$, for $j>i$, by

$$
v_{i, i}=1, \quad v_{i, j}=(a+(j-1)) v_{i, j-1} .
$$

Lemma 3.5. The coaction (2.7) on $z_{i}, i \in \mathbb{\square}_{0,3}$, is given, (for $n=0,1$ ) by

$$
\begin{gathered}
\delta\left(z_{2 n}\right)=\sum_{k=1}^{n} v_{k, n} x_{1} x_{21}^{n-k} g_{1}^{2 k-1} g_{2} \otimes z_{2 k-1}+\sum_{k=0}^{n} v_{k, n} x_{21}^{n-k} g_{1}^{2 k} g_{2} \otimes z_{2 k}, \\
\delta\left(z_{2 n+1}\right)=\sum_{k=0}^{n} v_{k, n+1} x_{1} x_{21}^{n-k} g_{1}^{2 k} g_{2} \otimes z_{2 k}+\sum_{k=0}^{n} v_{k+1, n+1} x_{21}^{n-k} g_{1}^{2 k+1} g_{2} \otimes z_{2 k+1} .
\end{gathered}
$$

Proof. Similar to the proof of [2, Lemma 4.2.5].

Lemma 3.5 implies that $K^{1}$ is of diagonal type with braiding given by

$$
c\left(z_{i} \otimes z_{j}\right)=q_{21}^{j-i} q_{22} z_{j} \otimes z_{i}, \quad \forall i, j .
$$

Now we are ready for to prove the main result of this Section.

Proof of Theorem 3.1. If $q_{22}=1$, then the Dynkin diagram of $K^{1}$ is totally disconnected with vertices labeled with 1 . Thus, if $a=1$, then $\operatorname{dim} \mathscr{B}\left(K^{1}\right)=2^{3}$ and $\operatorname{dim} \mathscr{B}\left(\mathfrak{L}_{\wp}(1,1)\right)=$ $2^{7}$; if $a \neq 1$, then $\operatorname{dim} \mathscr{B}\left(K^{1}\right)=2^{4}$ and $\operatorname{dim} \mathscr{B}\left(\mathfrak{L}_{\wp}(1, a)\right)=2^{8}$. If $q_{22} \neq 1$, then the Dynkin diagram of $K^{1}$ is

$$
a=1 \text { : }
$$

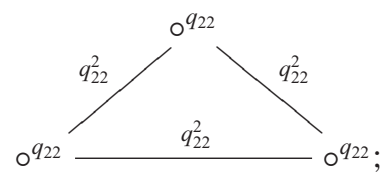

$a \neq 1:$

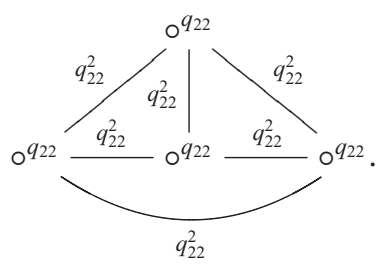

By inspection of the lists in $[\mathbf{1 0}, \mathbf{1 1}]$, we conclude that $\operatorname{dim} \mathscr{B}\left(K^{1}\right)=\infty$.

3.1. The presentation by generators and relations. Let $c$ be the braiding of $K^{1}$ as in (3.7). Then $q_{22}=1$ if and only if $c^{2}=\mathrm{id}$. Hence, for $a=1$ (resp. $\left.a \neq 1\right), \mathscr{B}\left(K^{1}\right)$ is the algebra generated by $z_{0}, z_{1}, z_{2}$ (resp. $z_{0}, z_{1}, z_{2}, z_{3}$ ) with relations:

$$
z_{i}^{2}=0, \quad z_{i} z_{j}=q_{21}^{j-i} z_{j} z_{i}, \quad i \neq j
$$

Thus, we have the following results. 
PROPOSITION 3.6. The algebra $\mathscr{B}\left(\mathfrak{L}_{\wp}(1,1)\right)$ is presented by generators $x_{1}, x_{2}, x_{3}$ with defining relations (2.5) and

$$
\begin{gathered}
x_{1} z_{j}=q_{12} z_{j} x_{1}, \quad z_{j+1}=x_{2} z_{j}+q_{12} z_{j} x_{2}, \quad j \in \mathbb{N}_{0}, \\
z_{i} z_{j}=q_{21}^{j-i} z_{j} z_{i}, \quad z_{j}^{2}=0, \quad z_{k}=0, \quad i, j \in \mathbb{\square}_{0,2}, \quad k \geq 3 .
\end{gathered}
$$

The dimension of $\mathscr{B}\left(\mathfrak{L}_{\wp}(1,1)\right)$ is $2^{7}$, since it has a PBW-basis:

$$
\left\{x_{1}^{m_{1}} x_{21}^{m_{2}} x_{2}^{m_{3}} z_{2}^{n_{2}} z_{1}^{n_{1}} z_{0}^{n_{0}}: m_{1}, m_{2}, n_{i} \in \mathbb{\square}_{0,1}, m_{3} \in \mathbb{\square}_{0,3}\right\} .
$$

PROpOSITION 3.7. The algebra $\mathscr{B}\left(\mathfrak{L}_{\wp}(1, a)\right), a \neq 1$, is presented by generators $x_{1}, x_{2}, x_{3}$ with defining relations (2.5) and

$$
\begin{gathered}
x_{1} z_{j}=\wp z_{j} x_{1}, \quad z_{j+1}=x_{2} z_{j}+\wp z_{j} x_{2}, \quad j \in \mathbb{N}_{0}, \\
z_{i} z_{j}=\wp^{i-j} z_{j} z_{i}, \quad z_{j}^{2}=0, \quad z_{k}=0, \quad i, j \in \mathbb{U}_{0,3}, \quad k \geq 4 .
\end{gathered}
$$

The dimension of $\mathscr{B}\left(\mathfrak{L}_{\wp}(1, a)\right)$ is $2^{8}$, since it has a PBW-basis:

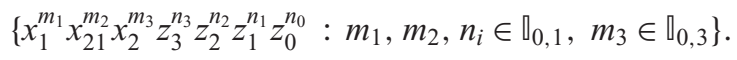

3.2. Realizations. Let $\left(g_{1}, \chi_{1}, \eta\right)$ be a YD-triple and $\left(g_{2}, \chi_{2}\right)$ a YD-pair for $H$, see Section 2.2.3. Let $(V, c)$ be a braided vector space with braiding (3.2). Then $\mathcal{V}_{g_{1}}\left(\chi_{1}, \eta\right) \oplus$ $\mathbb{k}_{g_{2}}^{\chi_{2}} \in{ }_{H}^{H} \mathcal{Y D}$ is a principal realization of $(V, c)$ over $H$ if

$$
q_{i j}=\chi_{j}\left(g_{i}\right), \quad \quad i, j \in \mathbb{\mathbb { V }}_{2} ; \quad a=q_{21}^{-1} \eta\left(g_{2}\right) .
$$

Thus, $(V, c) \simeq \mathcal{V}_{g_{1}}\left(\chi_{1}, \eta\right) \oplus \mathbb{k}_{g_{2}}^{\chi_{2}}$ as braided vector space. Hence, if $H$ is finite-dimensional and $(V, c) \simeq \mathfrak{L}_{\wp}(1, a), a \neq 0$, then $\mathscr{B}\left(\mathcal{V}_{g_{1}}\left(\chi_{1}, \eta\right) \oplus \mathbb{k}_{g_{2}}^{\chi_{2}}\right) \# H$ is a finite-dimensional Hopf algebra. Observe that the existence of a YD-triple for $H$ finite-dimensional is not granted, for instance, $\wp=q_{12}$ should be a root of 1 , otherwise there is no such triple. Suppose that ord $\wp=M \in \mathbb{N}$. Notice that $M$ is odd because char $\mathbb{k}=2$. Here are some explicit examples of finite-dimensional pointed Hopf algebras like this: take $\Gamma=\left\langle g_{1}\right\rangle \times\left\langle g_{2}\right\rangle$ where both $g_{1}$ and $g_{2}$ have order $2 M$. Then, $(V, c)$ is realized in ${ }_{\mathbb{k} \Gamma} \Gamma \mathcal{Y} \mathcal{D}$ with structure as in (3.3) and $\operatorname{dim} \mathscr{B}(V) \# \mathbb{k} \Gamma=2^{9} M^{2}$ (if $\left.a=1\right)$ or $2^{10} M^{2}$ (if $a \neq 1$ ).

4. One block and several points. Let $\theta \in \mathbb{N}_{\geq 3}, \square_{\theta}^{\dagger}=\rrbracket_{\theta} \cup\left\{\frac{3}{2}\right\}$; as usual $\lfloor x\rfloor$ is the integral part of $x \in \mathbb{R}$. We fix a matrix $\mathbf{q}=\left(q_{i j}\right)_{i, j \in \mathbb{l}_{\theta}}$ with entries in $\mathbb{k}^{\times}$and $\mathbf{a}=\left(1, a_{2}, \ldots, a_{\theta}\right) \in$ $\mathbb{k}^{\theta}$. We assume that

$$
q_{11}=1, \quad q_{1 j} q_{j 1}=1, \text { for all } j \in \mathbb{\square}_{2, \theta}, \quad \mathbf{a} \neq(1,0, \ldots, 0) .
$$

Let $(V, c)$ be the braided vector space of dimension $\theta+1$, with a basis $\left(x_{i}\right)_{i \in \square_{\theta}^{\dagger}}$ and braiding given by

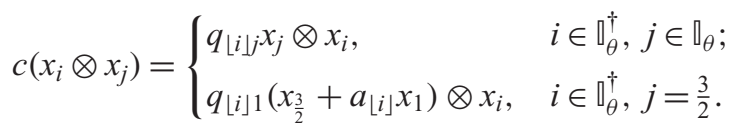

Then, $V=V_{1} \oplus V_{2}$ where $V_{1}=\left\langle x_{1}, x_{\frac{3}{2}}\right\rangle \simeq \mathcal{V}(1,2)$ (the block) and $V_{2}=\left\langle x_{2}, \ldots, x_{\theta}\right\rangle$ (the points). If $\Gamma=\mathbb{Z}^{\theta}$ with basis $\left(g_{h}\right)_{h \in \mathbb{U}_{\theta}}$, then $V$ can be realized in ${ }_{\mathbb{k} \Gamma}^{\mathbb{k} \Gamma} \mathcal{Y} \mathcal{D}$ as in (3.3). Here is the main result of this section. 
Theorem 4.1. Assume (4.1). Then $\operatorname{dim} \mathscr{B}(V)=\infty$.

We shall use the material from the previous section with $\frac{3}{2}$ replacing 2 for instance $x_{\frac{3}{2} 1}=x_{\frac{3}{2}} x_{1}+x_{1} x_{\frac{3}{2}}$. We shall apply the splitting technique cf. Section 2.3.2. To describe $K^{1}$, we introduce the elements:

$$
z_{i, n}:=\left(a d_{c} x_{\frac{3}{2}}\right)^{n} x_{i}, \quad \quad i \in \mathbb{\square}_{2, \theta}, \quad n \in \mathbb{N}_{0} .
$$

Let $i \in \mathbb{\square}_{2, \theta}, n \in \mathbb{N}_{0}$. By Lemma 3.2, we have that

$$
g_{1} \cdot z_{i, n}=q_{1 i} z_{i, n}, \quad z_{i, n+1}=x_{\frac{3}{2}} z_{i, n}+q_{1 i} z_{i, n} x_{\frac{3}{2}}, \quad x_{1} z_{i, n}=q_{1, i} z_{i, n} x_{1} .
$$

Consequently,

$$
g_{h} \cdot z_{i, n}=q_{h 1}^{n} q_{h i} z_{i, n}, \quad h \in \mathbb{\mathbb { V }}_{2, \theta} .
$$

In fact, $g_{h} \cdot z_{i, 0}=g_{h} \cdot x_{i}=q_{h i} x_{i}$. Suppose that $g_{h} \cdot z_{i, n}=q_{h 1}^{n} q_{h i} z_{i, n}$. Thus,

$$
\begin{aligned}
g_{h} \cdot z_{i, n+1} & =g_{h} \cdot\left(x_{\frac{3}{2}} z_{i, n}+q_{1 i} z_{i, n} x_{\frac{3}{2}}\right) \\
& =q_{h 1}\left(x_{\frac{3}{2}}+a_{h} x_{1}\right) q_{h 1}^{n} q_{h i} z_{i, n}+q_{1 i} q_{h 1}^{n+1} q_{h i} z_{i, n}\left(x_{\frac{3}{2}}+a_{h} x_{1}\right) \\
& =q_{h 1}^{n+1} q_{h i}\left(x_{\frac{3}{2}} z_{i, n}+q_{1 i} z_{i, n} x_{\frac{3}{2}}\right)=q_{h 1}^{n+1} q_{h i} z_{i, n+1} .
\end{aligned}
$$

As in Lemma 3.3, we define for $i \in \mathbb{\square}_{2, \theta}$,

$$
\begin{aligned}
& \mu_{0}^{(i)}=1, \quad \mu_{1}^{(i)}=a_{i}, \quad \mu_{2}^{(i)}=a_{i}, \quad \mu_{3}^{(i)}=a_{i}\left(a_{i}+1\right), \\
& y_{0}=1, \quad y_{1}=x_{1}, \quad y_{2}=x_{\frac{3}{2} 1}, \quad y_{3}=x_{1} x_{\frac{3}{2} 1} .
\end{aligned}
$$

Hence, $\partial_{h}\left(z_{i, n}\right)=0$ for $i \in \mathbb{\square}_{2, \theta}, n \in \mathbb{N}_{0}, i \neq h \in \mathbb{\square}_{\theta}^{\dagger}$ and

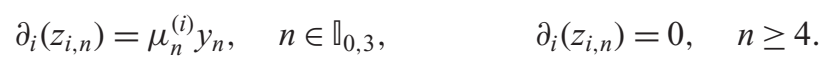

For $i \in \mathbb{Z}_{2, \theta}$, we define

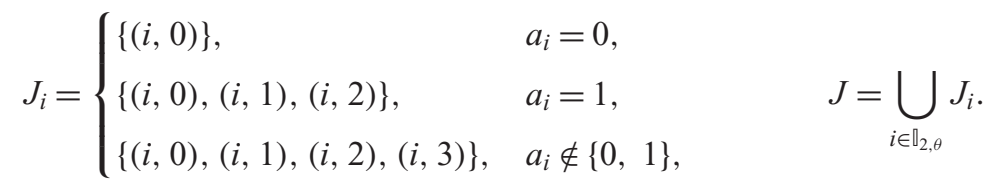

LEMMA 4.2. The family $B=\left(z_{i, n}\right)_{(i, n) \in J}$ is a basis of the braided vector space $K^{1}$, which is of diagonal type with braiding:

$$
c\left(z_{i, m} \otimes z_{j, n}\right)=q_{i 1}^{n} q_{1 j}^{m} q_{i j} z_{j, n} \otimes z_{i, m}, \quad \quad(i, m),(j, n) \in J .
$$

Proof. Arguing as in Lemma 3.4, we see that $B$ is a basis. We compute the coaction (2.7) on $z_{j, n}$ as in Lemma 3.5 and then (4.6) follows.

Proof of Theorem 4.1. It is enough to show that $\operatorname{dim} \mathscr{B}\left(K^{1}\right)=\infty$. By (4.6), we may assume that the Dynkin diagram of the matrix $\left(q_{i j}\right)_{i, j \in \mathbb{1}_{2, \theta}}$ is connected. We then may assume that $\theta=3$ by taking a suitable subdiagram; thus $\widetilde{q}_{23}:=q_{23} q_{32} \neq 0$. We distinguish then three cases. First assume $\mathrm{a}=(1, a, 0)$ with $a \neq 0$. By Theorem 3.1 applied to $V_{1} \oplus\left\langle x_{2}\right\rangle$, 
$q_{22}=1$. By Lemma $4.2, K^{1}$ is of diagonal type. If $a=1$, then its Dynkin diagram is

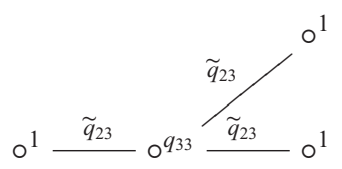

which does not appear in the list in [11]. If $a \neq 1$, then the diagram above appears a subdiagram. The case $\mathrm{a}=(1,0, b)$ with $b \neq 0$ is similar. As well, if $\mathrm{a}=(1, a, b)$ with $a, b \neq 0$, then the diagram above also appears a subdiagram of that of $K^{1}$.

5. Several blocks and one point. Let $t \geq 2$ and $\theta=t+1$. As in $[\mathbf{2}, 4]$ we use the notation:

$$
\square_{k}^{\ddagger}=\left\{k, k+\frac{1}{2}\right\}, \quad k \in \mathbb{\square}_{t} ; \quad \square^{\ddagger}=\square_{1}^{\ddagger} \cup \cdots \cup \square_{t}^{\ddagger} \cup\{\theta\} .
$$

We fix a matrix $\mathbf{q}=\left(q_{i j}\right)_{i, j \in \mathbb{Q}_{\theta}}$ with entries in $\mathbb{k}^{\times}$and $\mathbf{a}=\left(a_{1}, \ldots, a_{t}\right) \in \mathbb{k}^{t}$. We assume that

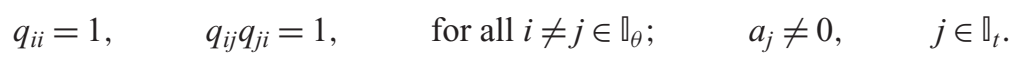

Let $\mathfrak{P}(\mathbf{q}, \mathbf{a})$ be the braided vector space with basis $\left(x_{i}\right)_{i \in \square^{\ddagger}}$ and braiding:

$$
c\left(x_{i} \otimes x_{j}\right)= \begin{cases}q_{\lfloor i\rfloor j\rfloor} x_{j} \otimes x_{i}, & \lfloor i\rfloor \leq t,\lfloor i\rfloor \neq\lfloor j\rfloor, \\ x_{j} \otimes x_{i}, & \lfloor i\rfloor=j \leq t, \\ \left(x_{j}+x_{\lfloor j\rfloor}\right) \otimes x_{i}, & \lfloor i\rfloor \leq t, j=\lfloor i\rfloor+\frac{1}{2}, \\ q_{\theta j} x_{j} \otimes x_{\theta}, & i=\theta, j \in \mathbb{\square}_{\theta}, \\ q_{\theta\lfloor j\rfloor}\left(x_{j}+a_{\lfloor j\rfloor} x_{\lfloor j\rfloor}\right) \otimes x_{\theta}, & i=\theta, j \notin \mathbb{\square}_{\theta} .\end{cases}
$$

Let $V_{1}=W_{1} \oplus \ldots \oplus W_{t}$ where $W_{k}=\left\langle x_{k}, x_{k+\frac{1}{2}}\right\rangle \simeq \mathcal{V}(1,2)$ (the blocks); and let $V_{2}=\left\langle x_{\theta}\right\rangle$ (the point). Then, $\mathfrak{P}(\mathbf{q}, \mathbf{a})=V_{1} \oplus V_{2}$. If $\Gamma=\mathbb{Z}^{\theta}$ with basis $\left(g_{i}\right)_{i \in \mathbb{V}_{\theta}}$, then there is an action of $\Gamma$ on $V$ determined by

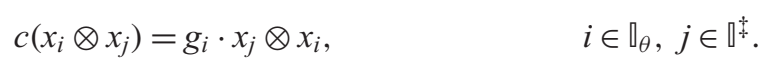

Thus, $V$ is realized in ${ }_{\mathbb{k} \Gamma}^{\mathrm{k} \Gamma} \mathcal{Y} \mathcal{D}$ with the grading $\operatorname{deg}\left(x_{i}\right)=g_{\lfloor i\rfloor}, i \in \square^{\ddagger}$.

Here is the main result of this section; see (5.9) for the explicit formula of the dimension.

Theorem 5.1. Assume (5.1). Then $\operatorname{dim} \mathscr{B}(\mathfrak{P}(\mathbf{q}, \mathbf{a}))<\infty$.

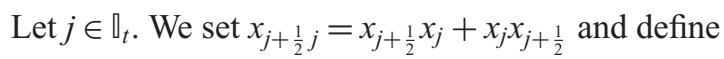

$$
\begin{aligned}
& \mu_{0}^{(j)}=1, \quad \mu_{1}^{(j)}=a_{j}, \quad \mu_{2}^{(j)}=a_{j}, \quad \mu_{3}^{(j)}=a_{j}\left(a_{j}+1\right), \quad \mu_{n}^{(j)}=0 \quad \text { if } n \geq 4, \\
& y_{j, 0}=1, \quad y_{j, 1}=x_{j}, \quad y_{j, 2}=x_{j+\frac{1}{2} j}, \quad y_{j, 3}=x_{j} x_{j+\frac{1}{2} j}, \quad y_{j, n}=0 \quad \text { if } n \geq 4 .
\end{aligned}
$$

To apply the splitting technique, see Section 2.3.2, we introduce the elements:

$$
щ_{\mathbf{n}}:=\left(\operatorname{ad}_{c} x_{\frac{3}{2}}\right)^{n_{1}} \ldots\left(\operatorname{ad}_{c} x_{t+\frac{1}{2}}\right)^{n_{t}} x_{\theta}, \quad \mathbf{n}=\left(n_{1}, \ldots, n_{t}\right) \in \mathbb{N}_{0}^{t} .
$$


We start establishing some useful formulas.

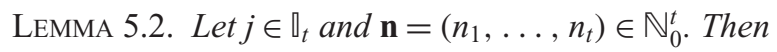

$$
\begin{gathered}
\operatorname{ad}_{c} x_{j}\left(щ_{\mathbf{n}}\right)=\operatorname{ad}_{c} x_{j+\frac{1}{2} j}\left(щ_{\mathbf{n}}\right)=0, \\
\operatorname{ad}_{c} x_{j+\frac{1}{2}}\left(щ_{\mathbf{n}}\right)=\prod_{i<j} q_{j i}^{n_{i}} 巛_{\mathbf{n}+\mathbf{e}_{j}}, \\
g_{j} \cdot щ_{\mathbf{n}}=q_{j \theta} \prod_{i=1}^{t} q_{j i}^{n_{i}}, \quad g_{\theta} \cdot щ_{\mathbf{n}}=\prod_{i=1}^{t} q_{\theta i}^{n_{i}} \amalg_{\mathbf{n}} \\
\partial_{j}\left(щ_{\mathbf{n}}\right)=\partial_{j+1}\left(щ_{\mathbf{n}}\right)=0, \quad \partial_{\theta}\left(щ_{\mathbf{n}}\right)=\prod_{i=1}^{t} \mu_{n_{i}}^{(i)} y_{1, n_{1}} \ldots y_{t, n_{t}} \cdot
\end{gathered}
$$

Proof. Similar to the proof of [2, Lemma 7.2.3].

Let us set

$$
b_{j}:=2 \text {, if } a_{j}=1, \quad b_{j}:=3, \text { if } a_{j} \neq 1, \quad \text { and } \mathbf{b}=\left(b_{1}, \ldots, b_{t}\right) \in \mathbb{N}^{t} .
$$

Arguing as in [2, Section 7.2], we conclude from Lemma 5.2:

Lemma 5.3. Let $\mathcal{A}=\left\{\mathbf{n} \in \mathbb{N}_{0}^{t}: \mathbf{n} \leq \mathbf{b}\right\}$ ordered lexicographically.

(i) The elements $\left(щ_{\mathbf{n}}\right)_{\mathbf{n} \in \mathcal{A}}$ form a basis of $K^{1}$.

(ii) The coaction (2.7) on $щ_{\mathbf{n}}$ is given by

$$
\delta\left(щ_{\mathbf{n}}\right)=\sum_{0 \leq \mathbf{k} \leq \mathbf{n}} v_{\mathbf{k}}^{\mathbf{n}} y_{1, n_{1}-k_{1}} \ldots y_{t, n_{t}-k_{t}} g_{1}^{k_{1}} \ldots g_{t}^{k_{t}} g_{\theta} \otimes щ_{\mathbf{k}}
$$

for some scalars $v_{\mathbf{k}}^{\mathbf{n}}, 0 \leq \mathbf{k} \leq \mathbf{n}$, with $v_{\mathbf{n}}^{\mathbf{n}}=1$.

(iii) The braided vector space $K^{1}$ is of diagonal type with respect to the basis $\left(щ_{\mathbf{n}}\right)_{\mathbf{n} \in \mathcal{A}}$ with matrix braiding $\left(p_{\mathbf{m}, \mathbf{n}}\right)_{\mathbf{m}, \mathbf{n} \in \mathcal{A}}$, where

$$
p_{\mathbf{m}, \mathbf{n}}=\prod_{i, j=1}^{t} q_{i j}^{m_{i} n_{j}} q_{i \theta}^{m_{i}} q_{\theta j}^{n_{j}} .
$$

Hence, the corresponding generalized Dynkin diagram has labels:

$$
p_{\mathbf{m}, \mathbf{m}}=1 \quad p_{\mathbf{m}, \mathbf{n}} p_{\mathbf{n}, \mathbf{m}}=1, \quad \mathbf{m} \neq \mathbf{n} .
$$

Proof of Theorem 5.1. By Lemma 5.3, $\operatorname{dim} \mathscr{B}\left(K^{1}\right)=2^{|\mathcal{A}|}$. Now the blocks $W_{i}$ and $W_{j}, i \neq j$, commute in the braided sense by definition, therefore $\mathscr{B}\left(V_{1}\right) \simeq$ $\mathscr{B}\left(W_{1}\right) \otimes \mathscr{B}\left(W_{2}\right) \ldots \otimes \mathscr{B}\left(W_{1}\right)$. Hence,

$$
\operatorname{dim} \mathscr{B}(\mathfrak{P}(\mathbf{q}, \mathbf{a}))=2^{4 t+|\mathcal{A}|} .
$$




\subsection{The presentation by generators and relations.}

Proposition 5.4. The algebra $\mathscr{B}(\mathfrak{P}(\mathbf{q}, \mathbf{a}))$ is presented by generators $x_{i}, i \in \square^{\ddagger}$, and relations:

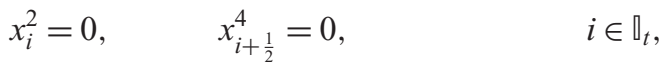

$$
\begin{aligned}
& x_{i+\frac{1}{2}}^{2} x_{i}+x_{i} x_{i+\frac{1}{2}}^{2}+x_{i} x_{i+\frac{1}{2}} x_{i}=0, \quad i \in \mathbb{\rrbracket}_{t},
\end{aligned}
$$

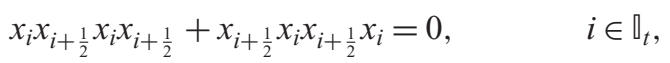

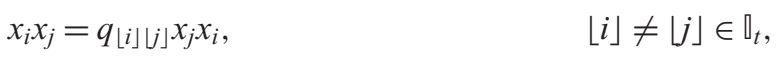

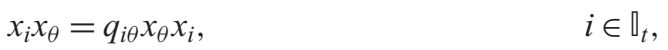

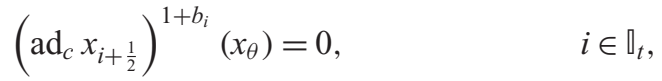

$$
\begin{aligned}
& щ_{\mathbf{m}} щ_{\mathbf{n}}=p_{\mathbf{m}, \mathbf{n}} щ_{\mathbf{n}} щ_{\mathbf{m}}, \quad \mathbf{m} \neq \mathbf{n} \in \mathcal{A}, \\
& щ_{\mathbf{n}}^{2}=0, \quad \mathbf{n} \in \mathcal{A} \text {. }
\end{aligned}
$$

A basis of $\mathscr{B}(\mathfrak{P}(\mathbf{q}, \mathbf{a}))$ is given by

$$
B=\left\{y_{1, m_{1}} x_{\frac{3}{2}}^{m_{2}} \ldots y_{t, m_{2 t-1}} x_{t+\frac{1}{2}}^{m_{2 t}} \prod_{\mathbf{n} \in \mathcal{A}} щ_{\mathbf{n}}^{b_{\mathbf{n}}}: 0 \leq b_{\mathbf{n}}<2,0 \leq m_{i}<4\right\} .
$$

Hence, $\operatorname{dim} \mathscr{B}(\mathfrak{P}(\mathbf{q}, \mathbf{a}))=2^{4 t+|\mathcal{A}|}$.

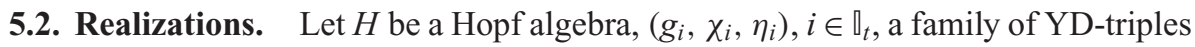
and $\left(g_{\theta}, \chi_{\theta}\right)$ a YD-pair for $H$, see Section 2.2.3. Let $(V, c)$ be a braided vector space with braiding (5.2). Then,

$$
\mathcal{V}:=\left(\oplus_{i \in \emptyset_{t}} \mathcal{V}_{g_{i}}\left(\chi_{i}, \eta_{i}\right)\right) \oplus \mathbb{k}_{g_{\theta}}^{\chi_{\theta}} \in_{H}^{H} \mathcal{Y} \mathcal{D}
$$

is a principal realization of $(V, c)$ over $H$ if

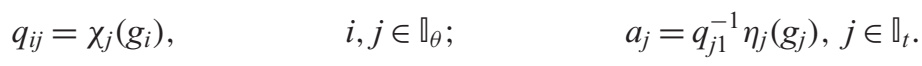

Consequently, if $H$ is finite-dimensional, then so is $\mathscr{B}(\mathcal{V}) \# H$. But the existence of such $H$ requires that all $q_{i j}$ 's are roots of 1 . In this case, let $\Gamma=(\mathbb{Z} / N)^{\theta}$ where $N$ is even and divisible by ord $q_{i j}$ for all $i, j$. Then, $(V, c)$ is realized in ${ }_{\mathbb{k} \Gamma}{ }^{\mathrm{Y}} \mathcal{Y} \mathcal{D}$ with action (5.3). Thus, $\mathscr{B}(\mathfrak{P}(\mathbf{q}, \mathbf{a})) \# \mathbb{k} \Gamma$ is a pointed Hopf algebra of dimension $2^{4 t+|\mathcal{A}|} N^{\theta}$.

6. One pale block and one point. An indecomposable Yetter-Drinfeld module which is decomposable as braided vector space is called a pale block [5]; the simplest examples were studied in $[2,4]$. We extend the analysis there to characteristic 2.

Let $\left(q_{i j}\right)_{i, j \in \mathbb{D}_{2}}$ be a matrix with nonzero entries; we assume that $q_{11}=1$ and $q_{12} q_{21}=1$; we set $\wp=q_{12}=q_{21}^{-1}$. Let $V=\mathfrak{E}_{\wp}\left(q_{22}\right)$ be the braided vector space of dimension 3 with basis $\left(x_{i}\right)_{i \in \mathbb{U}_{3}}$ and braiding given by 


$$
\left(c\left(x_{i} \otimes x_{j}\right)\right)_{i, j \in \mathbb{V}_{3}}=\left(\begin{array}{ccc}
x_{1} \otimes x_{1} & x_{2} \otimes x_{1} & q_{12} x_{3} \otimes x_{1} \\
x_{1} \otimes x_{2} & x_{2} \otimes x_{2} & q_{12} x_{3} \otimes x_{2} \\
q_{21} x_{1} \otimes x_{3} & q_{21}\left(x_{2}+x_{1}\right) \otimes x_{3} & q_{22} x_{3} \otimes x_{3}
\end{array}\right) .
$$

Let $V_{1}=\left\langle x_{1}, x_{2}\right\rangle$ (the pale block), $V_{2}=\left\langle x_{3}\right\rangle$ (the point), and $\Gamma=\mathbb{Z}^{2}$ with a basis $g_{1}, g_{2}$. Notice that $\mathscr{B}\left(V_{1}\right)$ is a truncated symmetric algebra of dimension 4 . We realize $V$ in ${ }_{\mathbb{k} \Gamma}^{\mathbb{k} \Gamma} \mathcal{Y}$ by $\operatorname{deg} x_{1}=\operatorname{deg} x_{2}=g_{1}, \operatorname{deg} x_{3}=g_{2}$ :

$$
\begin{array}{lll}
g_{1} \cdot x_{1}=x_{1}, & g_{1} \cdot x_{2}=x_{2}, & g_{1} \cdot x_{3}=q_{12} x_{3}, \\
g_{2} \cdot x_{1}=q_{21} x_{1}, & g_{2} \cdot x_{2}=q_{21}\left(x_{2}+x_{1}\right), & g_{2} \cdot x_{3}=q_{22} x_{3} .
\end{array}
$$

THEOREM 6.1. The Nichols algebra $\mathscr{B}\left(\mathfrak{E}_{\wp}\left(q_{22}\right)\right)$ is finite-dimensional if and only if $q_{22}=1$ or $q_{22}=\omega$, with $\omega \in \mathbb{G}_{3}^{\prime}$.

To apply the splitting technique, see Section 2.3.2, we introduce the elements:

$$
\amalg_{m, n}=\left(\operatorname{ad}_{c} x_{1}\right)^{m}\left(\operatorname{ad}_{c} x_{2}\right)^{n} x_{3}, \quad w_{m}=\amalg_{m, 0}, \quad z_{n}=\amalg_{0, n}, \quad m, n \in \mathbb{N}_{0} \text {. }
$$

By direct computation,

$$
\begin{array}{ll}
g_{1} \cdot \amalg_{m, n}=q_{12} \amalg_{m, n}, & g_{2} \cdot w_{m}=q_{21}^{m} q_{22} w_{m}, \\
z_{n+1}=x_{2} z_{n}+q_{12} z_{n} x_{2}, & \amalg_{m+1, n}=x_{1} \varpi_{m, n}+q_{12} \amalg_{m, n} x_{1}, \\
\partial_{1}\left(\amalg_{m, n}\right)=\partial_{2}\left(\amalg_{m, n}\right)=0, & \partial_{3}\left(w_{m}\right)=0, \text { for all } m>0 .
\end{array}
$$

Since $x_{1}$ and $x_{2}$ commute, $\varpi_{m, n}=\left(\operatorname{ad}_{c} x_{2}\right)^{n}\left(\varpi_{m, 0}\right)=\left(\operatorname{ad}_{c} x_{2}\right)^{n}\left(w_{m}\right)$. By $(6.5) w_{m}=0$ and thus $\amalg_{m, n}=0$, for all $m>0$. Hence, $\left\{z_{n}: n \in \mathbb{N}_{0}\right\}$ generates $K^{1}$. It is easy to check that

$$
g_{2} \cdot z_{n}=q_{21}^{n} q_{22} z_{n}, \quad \partial_{3}\left(z_{n}\right)=x_{1}^{n}, \quad n \in \mathbb{N}_{0} .
$$

As $x_{1}^{2}=0$, we conclude that $\left\{z_{0}, z_{1}\right\}$ is a basis of $K^{1}$. The coaction is given by $\delta\left(z_{0}\right)=$ $g_{2} \otimes z_{0}$ and $\delta\left(z_{1}\right)=x_{1} g_{2} \otimes z_{0}+g_{1} g_{2} \otimes z_{1}$. From (6.6) follows that $K^{1}$ is a braided vector space of diagonal type with braiding:

$$
c\left(z_{i} \otimes z_{j}\right)=q_{21}^{j-i} q_{22} z_{j} \otimes z_{i}, \quad i, j \in \mathbb{\square}_{0,1} .
$$

Proof of Theorem 6.1. If $q_{22}=1$, then the Dynkin diagram of $K^{1}$ is totally disconnected with vertices labelled with $q_{22}$. In this case, $z_{0}^{2}=z_{1}^{2}=0, \operatorname{dim} \mathscr{B}\left(K^{1}\right)=4$ and so $\operatorname{dim} \mathscr{B}\left(\mathfrak{E}_{\wp}(1)\right)=2^{4}$. If $q_{22} \neq 1$, the Dynkin diagram of $K^{1}$ is

$$
\circ^{q_{22}} \stackrel{q_{22}^{2}}{{ }^{q}}{ }^{22} \text {. }
$$

By inspection in the list of [9], $\operatorname{dim} \mathscr{B}\left(K^{1}\right)<\infty$ if and only if $q_{22}=\omega$, with $\omega \in \mathbb{G}_{3}^{\prime}$. In this case, $\operatorname{dim} \mathscr{B}\left(K^{1}\right)=3^{3}$ and so $\operatorname{dim} \mathscr{B}\left(\mathfrak{E}_{\wp}(\omega)\right)=2^{2} 3^{3}$.

\subsection{The presentation by generators and relations.}

Proposition 6.2. The algebra $\mathscr{B}\left(\mathfrak{E}_{\wp}(1)\right)$ is presented by generators $x_{1}, x_{2}, x_{3}$ with defining relations:

$$
\begin{array}{rlrl}
x_{1}^{2} & =0, & x_{2}^{2} & =0, \quad x_{1} x_{2}=x_{2} x_{1}, \\
x_{1} x_{3}=\wp x_{3} x_{1}, & z_{1}=x_{2} x_{3}+\wp x_{3} x_{2} \\
x_{3}^{2}=0, & z_{1}^{2}=0 .
\end{array}
$$


The dimension of $\mathscr{B}\left(\mathfrak{E}_{\wp}(1)\right)$ is $2^{4}$, since it has a PBW-basis:

$$
\left\{x_{1}^{m_{1}} x_{2}^{m_{2}} z_{1}^{n_{1}} x_{3}^{n_{0}}: m_{i}, n_{i} \in \mathbb{\square}_{0,1}\right\} .
$$

PROpOSITION 6.3. Let $z_{01}:=\operatorname{ad}_{c} x_{3}\left(z_{1}\right)$. The algebra $\mathscr{B}\left(\mathfrak{E}_{\wp}(\omega)\right)$ is presented by generators $x_{1}, x_{2}, x_{3}$ with defining relations:

$$
\begin{array}{cl}
x_{1}^{2}=0, & x_{2}^{2}=0, \quad x_{1} x_{2}=x_{2} x_{1}, \\
x_{1} x_{3}=\wp x_{3} x_{1}, & z_{1}=x_{2} x_{3}+\wp x_{3} x_{2} \\
x_{3}^{3}=0, & z_{1}^{3}=0 . \\
z_{01}^{3}=0, & \left(\operatorname{ad}_{c} x_{3}\right)^{2}\left(z_{1}\right)=0 .
\end{array}
$$

The dimension of $\mathscr{B}\left(\mathfrak{E}_{\wp}(\omega)\right)$ is $2^{2} 3^{3}$, since it has a PBW-basis:

$$
\left\{x_{1}^{m_{1}} x_{2}^{m_{2}} z_{1}^{n_{2}} z_{01}^{n_{1}} x_{3}^{n_{0}}: m_{i} \in \mathbb{\square}_{0,1}, n_{i} \in \mathbb{\square}_{0,2}\right\} .
$$

6.2. Realizations. Assume that $\wp$ is a root of 1 of odd order $M$. Take $\Gamma=\left\langle g_{1}\right\rangle \times$ $\left\langle g_{2}\right\rangle$ where $g_{1}$ has order $M$ and $g_{2}$ has order $2 M$. We realize $\mathfrak{E}_{\wp}(1)$ in ${ }_{\mathbb{k} \Gamma} \mathbb{V}^{2} \mathcal{D}$ by $\operatorname{deg} x_{1}=$ $\operatorname{deg} x_{2}=g_{1}, \operatorname{deg} x_{3}=g_{2}$ and action (6.2). Then, $\mathscr{B}\left(\mathfrak{E}_{\wp}(1)\right) \# \mathbb{k} \Gamma$ is a pointed Hopf algebra of dimension $2^{5} M^{2}$.

Also, let $\Upsilon=\left\langle h_{1}\right\rangle \times\left\langle h_{2}\right\rangle$ where $h_{1}$ has order $M$ and $h_{2}$ have order $P:=1 \mathrm{~cm}(6, M)$. We realize $\mathfrak{E}_{\wp}(\omega)$ in $\mathbb{k}_{\mathbb{k} \Upsilon}^{\mathbb{k} \Upsilon} \mathcal{Y D}$ by $\operatorname{deg} x_{1}=\operatorname{deg} x_{2}=h_{1}$, deg $x_{3}=h_{2}$ and action as in (6.2) with $h_{i}$ 's instead of the $g_{i}$ 's. Then, $\mathscr{B}\left(\mathfrak{E}_{\wp}(\omega)\right) \# \mathbb{k} \Upsilon$ is a pointed Hopf algebra of dimension $2^{3} 3^{3} M P$.

\section{REFERENCES}

1. N. Andruskiewitsch, An introduction to Nichols algebras, in Quantization, geometry and noncommutative structures in mathematics and physics (Cardona A., Morales P., Ocampo H., Paycha S. and Reyes A., Editors) (Springer International Publishing, 2017), 135-195.

2. N. Andruskiewitsch, I. Angiono and I. Heckenberger, On finite GK-dimensional Nichols algebras over abelian groups, Mem. Amer. Math. Soc. (to appear).

3. N. Andruskiewitsch, I. Angiono and I. Heckenberger, On finite GK-dimensional Nichols algebras of diagonal type, Contemp. Math. 728 (2019), 1-23.

4. N. Andruskiewitsch, I. Angiono and I. Heckenberger, Examples of finite-dimensional pointed Hopf algebras in positive characteristic, in Representation theory, mathematical physics and integrable systems, in honor of Nicolai Reshetikhin (Alexeev A., et al., Editor) (Progress in Mathematical, to appear).

5. N. Andruskiewitsch, I. Angiono and M. Moya Giusti, Nichols algebras of pale blocks (in preparation).

6. C. Cibils, A. Lauve and S. Witherspoon, Hopf quivers and Nichols algebras in positive characteristic, Proc. Amer. Math. Soc. 137(12) (2009), 4029-4041.

7. I. Heckenberger, Classification of arithmetic root systems, $A d v$. Math. 220 (2009), 59-124.

8. I. Heckenberger and H.-J Schneider, Yetter-Drinfeld modules over bosonizations of dually paired Hopf algebras, Adv. Math. 244 (2013), 354-394.

9. I. Heckenberger and J. Wang, Rank 2 Nichols algebras of diagonal type over fields of positive characteristic, SIGMA, Sym. Integrability Geom. Methods Appl. 11 (2015), Paper 011, 24 p.

10. J. Wang, Rank three Nichols algebras of diagonal type over arbitrary fields. Isr. J. Math. 218 (2017), 1-26.

11. J. Wang, Rank 4 finite-dimensional Nichols algebras of diagonal type in positive characteristic, J. Algebra 559 (2020), 547-579.

12. D. E. Radford, Hopf algebras, Series on Knots and Everything 49 (World Scientific, Hackensack, NJ, 2012), xxii+559. 